\title{
Effect of Different Dose and Application Methods of Paecilomyces lilacinus (Thom.) Samson against Root Knot Nematode, Meloidogyne incognita (Kofoidand White) Chitwood in Okra
}

\author{
Raja Kannan $^{1} \&$ Renganathan Veeravel ${ }^{1}$ \\ ${ }^{1}$ Department of Entomology, Annamalai University, Tamilnadu, India \\ Correspondence: Raja Kannan, Department of Entomology, Annamalai University, Tamilnadu, India. Tel: \\ 91-994-403-0789. E-mail:kaninsect@yahoo.co.in
}

Received: July18, 2012 Accepted: August 15, 2012 Online Published: October 12, 2012

doi:10.5539/jas.v4n11p119 URL: http://dx.doi.org/10.5539/jas.v4n11p119

\begin{abstract}
The biocontrol potential of Paecilomyces lilacinus was evaluated in field conditions in two seasons during 2005-2008 at Annamalainagar and Vallambadugai of Cuddalore district of Tamilnadu, India. In the field evaluation of different doses and application methods of P. lilacinus viz., seed treatment, seedling treatments, soil application treatments and the integration treatments produced mixed results on the growth of okra in both Annamalainagar and Vallambadugai. In two field trials of okra at two locations, shoot length, shoot weight and root length were significantly increased in mixture treatments compared to individual treatments, principally combination of seedling treatment $(10 \mathrm{~g} / \mathrm{l}$ water $)+$ soil application treatment $(5.0 \mathrm{~kg} / \mathrm{acre})$ documented maximum shoot length (60 and 90 DAS), shoot weight (90 DAS) and root length (90 DAS) and they were positively correlated with fruit yield of okra.
\end{abstract}

Keywords: Abelmoschus esculentus, root knot nematode, biological control, Paecilomyces lilacinus

\section{Introduction}

Okra (Abelmoschus esculentus (L.)Moench) is an important vegetable in Indian cuisine (Jain et al., 2007), which has been ravaged by many nematodes and among them root knot nematode have been reported as a major problem. Meloidogyne incognita (Kofoid and White) Chitwood was prevalent in okra and it occupied relatively high proportion in soil and rootsand their presence in rhizosphere soil lead to serious infestation and caused reduction in fruit yield ranging from 31.04 to 61.0 per cent (Swarup, 1962; Sivakumar et al., 1976; A. A. Khan \& M. W. Khan, 1990; Mojumder et al., 2004). Among different opportunistic soil Hypomycetes, Paecilomyces lilacinus (Thom). Samson was found to be capable of parasitizing both the stages of eggs and larvae of Meloidogyne spp. and might become a potential eco-friendly biological control agent in the management of plant parasitic nematodes (Cabanillasand \& Barker, 1989). Many researchers reported the effectiveness of $P$. lilacinus in controlling Meloidogyne spp. in different crops (Devrajan \& Rajendran, 2002; Ahmad \& Khan, 2004; Brand et al., 2004; Kiewnick \& Sikora, 2006).

First report of a successful application of biocontrol agent for $M$. incognita under field application was made by Jatala et al. (1980). Plants grown in plots inoculated with fungus had significantly lower root gall index than those grown in plots applied with organic matter and nematicides. Eighty six per cent of egg masses collected from the plants grown in the fungus plots were infected with $P$. lilacinus and 54 percent of the eggs were destroyed. A field experiment to study the effect of castor leaves and P. lilacinus alone and in combination on $M$. incognita infecting tomato revealed reduction in nematode population i.e., gall index, juvenile and females and increased plant growth. The combination treatment resulted with 86 and 100 per cent reduction in gall index and juvenile population respectively with control. The registered shoot length (ranged from 16.00 to $37.25 \mathrm{~cm}$ ) and fresh shoot weight (ranged 2.12 to $26.62 \mathrm{~g}$ ) were at the maximum in best treatment and minimum in control (Zaki \& Bhatti, 1999). Investigations on the biocontrol of different nematodes associated with chickpea in field conditions using different oil cakes viz., neem, castor, groundnut, linseed and sunflower @ $110 \mathrm{~kg} \mathrm{~N} / \mathrm{ha}$ and $P$. lilacinus in alone and in combination by Tiyagi and Ajaz (2004) revealed significant improvement in plant growth parameters (plant weight, pollen fertility, pod numbers and chlorophyll content) and reduction in the population of plant parasitic nematodes. Neem cake in the presence of $P$. lilacinus brought about highest improvement in shoot weight, root weight, pods/ plant and chlorophyll compared to neem cake alone whereas 
this combination reduced the galls formation from 55.0 to 42.1 in neem alone treatment. Hasan (2004) conducted a field trial to investigate the effect of P. lilacinus as seed treatment alone or in combination with three kinds of organic matter and a nematicide on $M$. incognita infecting cowpea. Combination of seed treatment with soil application of fungus into infested soil one week prior to sowing found to be more effective in reducing the root knot incidence and increasing the forage yield than when used alone. In this context, an attempt has been made to evaluate different dose and application methods of $P$. lilacinus against $M$. incognita in okra under field conditions.

\section{Method}

The biocontrol potential of P. lilacinus was evaluated in field conditions in two seasons during 2005-2008 at Annamalainagar and Vallambadugai. The fungal spores pounded on sorghum grains were used as the source of fungal inoculum and they were used in the field inoculation studies. The spore load in the pounded sorghum was estimated and based on the spore load the following treatments were fixed for the field evaluation of $P$. lilacinus against root knot nematode in okra.

$\begin{array}{llll}\mathrm{T}_{1} & = & \text { P. lilacinus } & \text { seed treatment } 5 \mathrm{~g} / \mathrm{Kg} \text { seeds } \\ \mathrm{T}_{2} & = & \text { P. lilacinus } & \text { seedling dip } 5 \mathrm{~g} / \mathrm{l} \text { water } \\ \mathrm{T}_{3} & = & \text { P. lilacinus } & \text { seedling dip } 10 \mathrm{~g} / \mathrm{l} \text { water } \\ \mathrm{T}_{4} & = & \text { P. lilacinus } & \text { soil application } @ 2.5 \mathrm{~kg} / \mathrm{acre}+450 \mathrm{~g} \text { neem cake at } 45 \text { DAS } \\ \mathrm{T}_{5} & = & \text { P. lilacinus } & \text { soil application } @ 5.0 \mathrm{~kg} / \mathrm{acre}+450 \text { g neem cake at } 45 \text { DAS } \\ \mathrm{T}_{6} & = & \mathrm{T}_{1}+\mathrm{T}_{4} \\ \mathrm{~T}_{7} & = & \mathrm{T}_{2}+\mathrm{T}_{4} \\ \mathrm{~T}_{8} & = & \mathrm{T}_{3}+\mathrm{T}_{4} \\ \mathrm{~T}_{9} & = & \mathrm{T}_{1}+\mathrm{T}_{5} \\ \mathrm{~T}_{10} & = & \mathrm{T}_{2}+\mathrm{T}_{5} \\ \mathrm{~T}_{11} & = & \mathrm{T}_{3}+\mathrm{T}_{5} \\ \mathrm{~T}_{12} & = & \text { Control }\end{array}$

The fungal spore population $\left(1 \times 10^{8}\right)$ was maintained and applied uniformly in all vegetable crops under field conditions to avoid bias in experiment.

The field was ploughed twice, clods were removed, pulverized and based on the lay out ridges and furrows were prepared and the treatment plots were assigned based on lottery method. Surface sterilized okra seeds (with $1 \%$ $\mathrm{NaOCl}$ ) was sown (two seeds) in $3.0 \times 2.4 \mathrm{~m}$ plots with a spacing of $60 \times 45 \mathrm{~cm}$ in a field infested with M. incognita with initial population of 342.67 juveniles (100 cc soil) in the first trial and 302.67 Juveniles (100 cc soil) in the second trial. The seed treatment was given to the seeds before sowing and sown according to treatment order. Different doses of fungus were prepared from fungus inoculated sorghum grains and the treatments were given as per schedule. For soil drench treatment in okra, $5 \mathrm{~g}\left(\mathrm{~T}_{2}\right)$ and $10 \mathrm{~g}\left(\mathrm{~T}_{3}\right)$ pounded sorghum grains were mixed with one litre of water, and prepared concentrations were applied as soil drenching near the root zone of the okra plant after 15 days of sowing as per treatment order (Khan et al., 1985). Regular practices of crop production were carried out.

Data on plant height (Initial, 60 DAS), soil nematode population (60 DAS) and yield was recorded at crop stand. The plants were uprooted after harvest ( 90 days after sowing) and the data on plant height, fresh shoot weight, fresh root length and root weight, root gall numbers/plant, root gall index, soil nematode population (100 cc soil), number of egg mass/g root and egg mass parasitization were recorded and calculated.

\subsection{Statistical Analysis}

The data on field experiments laid with completely randomized design were analyzed statistically and based on the analysis of variance (ANOVA), treatment means were compared and ranked following least significant difference test (LSD) (Gomez \& Gomez, 1976; Rangaswamy, 1995). 


\section{Results and Discussion}

\subsection{Field Trial I}

The integration of seedling treatment with soil application treatment $\left(\mathrm{T}_{11}\right)$ produced maximum shoot length $(44.67 \mathrm{~cm})$ after 60 days of sowing followed by $\mathrm{T}_{10}(44.33 \mathrm{~cm}), \mathrm{T}_{9}(44.00 \mathrm{~cm}), \mathrm{T}_{8}(43.33 \mathrm{~cm}), \mathrm{T}_{7}(42.33 \mathrm{~cm})$ and $\mathrm{T}_{6}(42.00 \mathrm{~cm})$ and they differ significantly. Ninety days of sowing investigations revealed maximum $(51.00 \mathrm{~cm})$ of shoot length in a combination treatment $T_{10}$ and minimum in control $(44.33 \mathrm{~cm})$. Shoot weight of okra assessed after plant removal at 90 days of sowing revealed that the values ranged between 36.67 and $51.33 \mathrm{~g}$ with maximum in a combination treatment $\mathrm{T}_{11}(51.33 \mathrm{~g})$ and minimum in individual seedling treatment $\left(\mathrm{T}_{3}\right)(36.67 \mathrm{~g})$. Treatment combination ( $\left.\mathrm{T}_{11}\right)$ involving $\mathrm{T}_{3}$ and $\mathrm{T}_{5}$ produced maximum shoot weight $(51.33 \mathrm{~g})$ followed by $\mathrm{T}_{10}$ (48.67 g), $\mathrm{T}_{8}(45.67 \mathrm{~g}), \mathrm{T}_{9}(45.00 \mathrm{~g})$ with significant difference among them (Table 1).

Table1. Influence of different application methods of P. lilacinus on on the growth of okra infested with $\quad M$. incognita at Annamalainagar

\begin{tabular}{|c|c|c|c|c|c|c|c|c|c|c|c|}
\hline \multirow[b]{2}{*}{ Treatments } & \multicolumn{5}{|c|}{ Shoot length $(\mathrm{cm})$} & \multicolumn{2}{|c|}{ Shoot weight (g) } & \multicolumn{2}{|c|}{ Root length $(\mathrm{cm})$} & \multicolumn{2}{|c|}{ Root weight } \\
\hline & $\begin{array}{c}15 \\
\text { DAS }\end{array}$ & 60 DAS & $\begin{array}{c}\% \uparrow \downarrow \\
\text { OC }\end{array}$ & 90 DAS & $\begin{array}{c}\% \uparrow \downarrow \\
\text { OC }\end{array}$ & 90 DAS & $\begin{array}{c}\% \uparrow \downarrow \\
\text { OC }\end{array}$ & 90 DAS & $\begin{array}{c}\% \uparrow \downarrow \\
\text { OC }\end{array}$ & 90 DAS & $\begin{array}{c}\% \uparrow \downarrow \\
\text { OC }\end{array}$ \\
\hline $\mathrm{T}_{1}$ & $\begin{array}{c}15.33 \\
\pm 0.58\end{array}$ & $\begin{array}{c}39.67 \pm \\
1.15^{\mathrm{g}}\end{array}$ & 3.49 & $\begin{array}{c}45.00 \pm \\
2.00^{\mathrm{d}}\end{array}$ & 1.51 & $\begin{array}{c}37.00 \pm \\
2.65^{\mathrm{g}}\end{array}$ & -7.50 & $\begin{array}{c}20.33 \pm \\
0.58^{\mathrm{h}}\end{array}$ & -6.17 & $\begin{array}{c}16.67 \pm \\
1.15^{\mathrm{abc}}\end{array}$ & -7.41 \\
\hline $\mathrm{T}_{2}$ & $\begin{array}{c}15.33 \\
\pm 0.29\end{array}$ & $\begin{array}{c}40.33 \pm \\
0.58^{\mathrm{fg}}\end{array}$ & 5.23 & $\begin{array}{c}46.33 \pm \\
0.58^{\mathrm{cd}}\end{array}$ & 4.52 & $\begin{array}{c}37.67 \pm \\
3.51^{\mathrm{g}}\end{array}$ & -5.83 & $\begin{array}{c}21.67 \pm \\
0.58^{\mathrm{gh}}\end{array}$ & 0.00 & $\begin{array}{c}17.00 \pm \\
1.00^{\mathrm{ab}}\end{array}$ & -5.56 \\
\hline $\mathrm{T}_{3}$ & $\begin{array}{c}15.00 \\
\pm 0.00\end{array}$ & $\begin{array}{c}40.67 \pm \\
0.58^{\mathrm{fg}}\end{array}$ & 6.10 & $\begin{array}{c}45.00 \pm \\
1.00^{\mathrm{d}}\end{array}$ & 1.51 & $\begin{array}{c}36.67 \pm \\
3.51^{\mathrm{g}}\end{array}$ & -8.33 & $\begin{array}{c}22.33 \pm \\
1.53^{\mathrm{g}}\end{array}$ & 3.06 & $\begin{array}{c}16.67 \pm \\
0.58^{\mathrm{abc}}\end{array}$ & -7.41 \\
\hline $\mathrm{T}_{4}$ & $\begin{array}{c}15.33 \\
\pm 0.29\end{array}$ & $\begin{array}{c}41.00 \pm \\
0.00^{\mathrm{ef}}\end{array}$ & 6.97 & $\begin{array}{c}48.00 \pm \\
1.00^{\mathrm{bc}}\end{array}$ & 8.28 & $\begin{array}{c}38.67 \pm \\
0.58^{\mathrm{fg}}\end{array}$ & -3.33 & $\begin{array}{c}23.33 \pm \\
0.58^{\mathrm{fg}}\end{array}$ & 7.68 & $\begin{array}{c}18.33 \pm \\
0.58^{\mathrm{a}}\end{array}$ & 1.85 \\
\hline $\mathrm{T}_{5}$ & $\begin{array}{c}15.17 \\
\pm 0.29\end{array}$ & $\begin{array}{c}41.33 \pm \\
0.58^{\text {def }}\end{array}$ & 7.84 & $\begin{array}{c}48.33 \pm \\
1.53^{\mathrm{bc}}\end{array}$ & 9.03 & $\begin{array}{c}41.67^{\mathrm{f}} \pm \\
0.58^{\mathrm{de}}\end{array}$ & 4.17 & $\begin{array}{c}25.67 \pm \\
0.58^{\mathrm{de}}\end{array}$ & 18.44 & $\begin{array}{l}16.00 \pm \\
1.00^{\mathrm{bcd}}\end{array}$ & -11.11 \\
\hline $\mathrm{T}_{6}$ & $\begin{array}{c}15.17 \\
\pm 0.29\end{array}$ & $\begin{array}{c}42.00 \pm \\
1.00^{\mathrm{de}}\end{array}$ & 9.57 & $\begin{array}{c}49.33 \pm \\
1.15^{\mathrm{ab}}\end{array}$ & 11.29 & $\begin{array}{c}41.33 \pm \\
1.53^{\mathrm{ef}}\end{array}$ & 3.33 & $\begin{array}{c}26.67 \pm \\
0.58^{\text {cde }}\end{array}$ & 23.06 & $\begin{array}{c}15.00 \pm \\
1.00^{\mathrm{cd}}\end{array}$ & -16.67 \\
\hline $\mathrm{T}_{7}$ & $\begin{array}{c}15.33 \\
\pm 0.29\end{array}$ & $\begin{array}{c}42.33 \pm \\
0.58^{\mathrm{cd}}\end{array}$ & 10.44 & $\begin{array}{c}49.33 \pm \\
0.58^{\mathrm{ab}}\end{array}$ & 11.29 & $\begin{array}{l}43.00 \pm \\
2.00^{\text {cde }}\end{array}$ & 7.50 & $\begin{array}{c}25.00 \pm \\
1.00^{\mathrm{ef}}\end{array}$ & 15.37 & $\begin{array}{c}16.67 \pm \\
0.58^{\mathrm{abc}}\end{array}$ & -7.41 \\
\hline $\mathrm{T}_{8}$ & $\begin{array}{c}15.17 \\
\pm 0.29\end{array}$ & $\begin{array}{c}43.33 \pm \\
0.58^{\mathrm{bc}}\end{array}$ & 13.05 & $\begin{array}{c}49.00 \pm \\
1.00^{\mathrm{ab}}\end{array}$ & 10.53 & $\begin{array}{c}45.67 \pm \\
0.58^{\mathrm{bc}}\end{array}$ & 14.17 & $\begin{array}{c}27.33 \pm \\
0.58^{\text {bcd }}\end{array}$ & 26.13 & $\begin{array}{c}14.67 \pm \\
0.58^{d}\end{array}$ & -18.52 \\
\hline $\mathrm{T}_{9}$ & $\begin{array}{c}15.33 \\
\pm 0.29\end{array}$ & $\begin{array}{c}44.00 \pm \\
1.00^{\mathrm{ab}}\end{array}$ & 14.79 & $\begin{array}{c}49.00 \pm \\
1.00^{\mathrm{ab}}\end{array}$ & 10.53 & $\begin{array}{c}45.00 \pm \\
2.00^{\mathrm{cd}}\end{array}$ & 12.50 & $\begin{array}{c}28.00 \pm \\
1.00^{\mathrm{abc}}\end{array}$ & 29.21 & $\begin{array}{l}16.00 \pm \\
1.00^{\mathrm{bcd}}\end{array}$ & -11.11 \\
\hline $\mathrm{T}_{10}$ & $\begin{array}{c}15.17 \\
\pm 0.29\end{array}$ & $\begin{array}{c}44.33 \pm \\
0.58^{\mathrm{ab}}\end{array}$ & 15.66 & $\begin{array}{c}51.00 \pm \\
1.00^{\mathrm{a}}\end{array}$ & 15.05 & $\begin{array}{c}48.67 \pm \\
2.89^{\mathrm{ab}}\end{array}$ & 21.67 & $\begin{array}{c}29.00 \pm \\
1.73^{\mathrm{ab}}\end{array}$ & 33.83 & $\begin{array}{l}16.00 \pm \\
1.00^{\mathrm{bcd}}\end{array}$ & -11.11 \\
\hline $\mathrm{T}_{11}$ & $\begin{array}{c}15.33 \\
\pm 0.29\end{array}$ & $\begin{array}{c}44.67 \pm \\
1.15^{\mathrm{a}}\end{array}$ & 16.53 & $\begin{array}{c}50.00 \pm \\
1.00^{\mathrm{ab}}\end{array}$ & 12.79 & $\begin{array}{c}51.33 \pm \\
2.31^{\mathrm{a}}\end{array}$ & 28.33 & $\begin{array}{c}29.67 \pm \\
1.53^{\mathrm{a}}\end{array}$ & 36.90 & $\begin{array}{c}18.33 \pm \\
1.53^{\mathrm{a}}\end{array}$ & 1.85 \\
\hline $\mathrm{T}_{12}$ & $\begin{array}{c}15.33 \\
\pm 0.29\end{array}$ & $\begin{array}{c}38.33 \pm \\
0.58^{\mathrm{h}}\end{array}$ & & $\begin{array}{c}44.33 \pm \\
2.52^{\mathrm{d}}\end{array}$ & & $\begin{array}{c}40.00 \pm \\
1.00^{\mathrm{g}}\end{array}$ & & $\begin{array}{c}21.67 \pm \\
2.08^{\mathrm{h}}\end{array}$ & & $\begin{array}{c}18.00 \pm \\
1.73^{\mathrm{a}}\end{array}$ & \\
\hline S. $E m \pm$ & NS & 0.586 & & 1.082 & & 1.645 & & 0.846 & & 0.822 & \\
\hline $\begin{array}{c}\text { C.D. } \\
(P=0.05)\end{array}$ & & 1.215 & & 2.243 & & 3.411 & & 1.754 & & 1.704 & \\
\hline
\end{tabular}

Each value is an average of three replicates; in a column, means followed by common letter(s) are not significantly different $(P=0.05)$ by Least Significant Difference Test; DAP-Days after planting; \% $\uparrow \downarrow$ OC-per cent increase or decrease over control; NS-No significance at $P=0.05 ; \mathrm{T}_{1}-P$. lilacinus seed treatment, $\mathrm{T}_{2}-P$. lilacinus seedling treatment $(5 \mathrm{~g} / 1$ water $) . \mathrm{T}_{3}-P$. lilacinus seedlingtreatment $(10 \mathrm{~g} / 1$ water $), \mathrm{T}_{4}-P$. lilacinus soil application (2.5 kg/acre), $\mathrm{T}_{5}-P$. lilacinus soil application (5.0 kg/acre), $\mathrm{T}_{6}-\mathrm{T}_{1}+\mathrm{T}_{4}, \mathrm{~T}_{7}-\mathrm{T}_{2}+\mathrm{T}_{4}, \mathrm{~T}_{8}-\mathrm{T}_{3}+\mathrm{T}_{4}, \mathrm{~T}_{9}-\mathrm{T}_{1}+\mathrm{T}_{5}$, $\mathrm{T}_{10}-\mathrm{T}_{2}+\mathrm{T}_{5}, \mathrm{~T}_{11}-\mathrm{T}_{3}+\mathrm{T}_{5}$; and $\mathrm{T}_{12}$-Control. 
Table 2.Effect of different application methods of $P$. lilacinus on population density and gall formation of $M$. incognita in okra at Annamalainagar

\begin{tabular}{|c|c|c|c|c|c|c|c|c|}
\hline \multirow{2}{*}{ Treatments } & \multicolumn{4}{|c|}{ Juvenile population (100 cc soil) } & \multicolumn{2}{|c|}{ Galls/plant } & \multicolumn{2}{|c|}{ Females/g root } \\
\hline & $60 \mathrm{DAS}$ & $\% \uparrow \downarrow \mathrm{OC}$ & $90 \mathrm{DAS}$ & $\% \uparrow \downarrow \mathrm{OC}$ & $90 \mathrm{DAS}$ & $\% \uparrow \downarrow \mathrm{OC}$ & $90 \mathrm{DAS}$ & $\% \uparrow \downarrow \mathrm{OC}$ \\
\hline $\mathrm{T}_{1}$ & $318.67 \pm 4.16^{\mathrm{fg}}$ & -3.04 & $213.67 \pm 2.08^{\mathrm{g}}$ & -1.84 & $26.00 \pm 2.65^{\mathrm{def}}$ & -33.33 & $23.00 \pm 2.00^{\mathrm{g}}$ & -14.81 \\
\hline $\mathrm{T}_{2}$ & $306.00 \pm 3.61^{\mathrm{f}}$ & -6.90 & $196.00 \pm 2.65^{\text {ef }}$ & -9.96 & $28.67 \pm 2.52^{\mathrm{e}}$ & -26.50 & $20.00 \pm 1.00^{\mathrm{f}}$ & -25.93 \\
\hline $\mathrm{T}_{3}$ & $312.67 \pm 3.06^{\mathrm{f}}$ & -4.87 & $194.67 \pm 1.53^{\mathrm{e}}$ & -10.57 & $24.33 \pm 5.03^{\text {cde }}$ & -37.61 & $18.33 \pm 2.08^{\mathrm{f}}$ & -32.10 \\
\hline $\mathrm{T}_{4}$ & $280.67 \pm 5.03^{\mathrm{e}}$ & -14.61 & $202.33 \pm 3.21^{\mathrm{f}}$ & -7.05 & $22.67 \pm 3.06^{\mathrm{bcd}}$ & -41.88 & $16.00 \pm 1.00^{\mathrm{e}}$ & -40.74 \\
\hline $\mathrm{T}_{5}$ & $273.33 \pm 3.06^{\mathrm{e}}$ & -16.84 & $192.33 \pm 5.86^{\mathrm{e}}$ & -11.64 & $20.67 \pm 1.53^{\mathrm{bc}}$ & -47.01 & $15.33 \pm 1.15^{\mathrm{e}}$ & -43.21 \\
\hline $\mathrm{T}_{6}$ & $269.67 \pm 4.16^{\mathrm{de}}$ & -17.95 & $181.33 \pm 4.51^{\mathrm{d}}$ & -16.69 & $22.00 \pm 2.65^{\mathrm{bcd}}$ & -43.59 & $14.67 \pm 0.58^{\mathrm{de}}$ & -45.68 \\
\hline $\mathrm{T}_{7}$ & $254.33 \pm 5.13^{\mathrm{bc}}$ & -22.62 & $176.33 \pm 2.52^{\mathrm{cd}}$ & -18.99 & $20.67 \pm 1.53^{\mathrm{bc}}$ & -47.01 & $14.00 \pm 1.00^{\text {cde }}$ & -48.15 \\
\hline $\mathrm{T}_{8}$ & $254.00 \pm 6.24^{\mathrm{bc}}$ & -22.72 & $175.33 \pm 5.69^{\mathrm{cd}}$ & -19.45 & $19.00 \pm 2.00^{\mathrm{b}}$ & -51.28 & $13.00 \pm 1.00^{\mathrm{bcd}}$ & -51.85 \\
\hline $\mathrm{T}_{9}$ & $259.67 \pm 9.50^{\mathrm{cd}}$ & -20.99 & $172.33 \pm 2.52^{\mathrm{bc}}$ & -20.83 & $18.67 \pm 2.08^{b}$ & -52.14 & $12.33 \pm 0.58^{\mathrm{bc}}$ & -54.32 \\
\hline $\mathrm{T}_{10}$ & $245.33 \pm 5.69^{\mathrm{ab}}$ & -25.36 & $166.00 \pm 4.58^{\mathrm{b}}$ & -23.74 & $18.33 \pm 2.52^{\mathrm{b}}$ & -52.99 & $11.00 \pm 1.00^{\mathrm{ab}}$ & -59.26 \\
\hline $\mathrm{T}_{11}$ & $240.67 \pm 9.02^{\mathrm{a}}$ & -26.78 & $156.33 \pm 4.04^{\mathrm{a}}$ & -28.18 & $13.67 \pm 3.21^{\mathrm{a}}$ & -64.96 & $9.33 \pm 0.58^{\mathrm{a}}$ & -65.43 \\
\hline $\mathrm{T}_{12}$ & $328.67 \pm 15.63^{\mathrm{g}}$ & & $217.67 \pm 6.66^{\mathrm{g}}$ & & $39.00 \pm 3.00^{\mathrm{f}}$ & & $27.00 \pm 2.00^{\mathrm{h}}$ & \\
\hline S. $E m \pm$ & 0.176 & & 0.119 & & 0.252 & & 1.061 & \\
\hline $\begin{array}{c}\text { C.D. } \\
(P=0.05)\end{array}$ & 0.366 & & 0.247 & & 0.523 & & 2.201 & \\
\hline
\end{tabular}

Initial juvenile population-342.67/100 cc soil; each value is an average of three replicates; in a column, means followed by common letter(s) are not significantly different $(\mathrm{P}=0.05)$ by Least Significant Difference Test; DAP-Days after planting; \% $\uparrow \downarrow$ OC-per cent increase or decrease over control; T1-P. lilacinus seed treatment, T2-P. lilacinus seedling treatment ( $5 \mathrm{~g} / 1$ water). T3-P. lilacinus seedling treatment (10 g/l water), T4-P. lilacinus soil application (2.5 kg/acre), T5-P. lilacinus soil application (5.0 kg/acre), T6-T1+T4, T7-T2+T4, T8-T3+T4, T9 -T1+T5, T10-T2+T5, T11-T3+T5; and T12-Control

Combination of seedling treatment $\left(\mathrm{T}_{3}\right)$ and soil application treatment $\left(\mathrm{T}_{5}\right)$ recorded the maximum root length $\left(T_{11}\right)(29.67 \mathrm{~cm})$ which was significantly superior over other treatments. Results on root weight even though disclosed statistical significance among treatments, resulted with an irregular increase or decrease in root weights among independent treatments. The same trend of irregular root weight was manifested with combination treatments also with significant difference, wherein the maximum root weight was noticed in the maximum P. lilacinus inoculated treatment $\mathrm{T}_{11}(18.33 \mathrm{~g})$ (Table 1).

All treatments, alone or partnered exerted distinct influence in reducing the juvenile population. Among associated treatments, maximum population was evidenced in $\mathrm{T}_{6}$ (269.97) and minimum population was registered by $\mathrm{T}_{11}$ (240.67) which significantly reduced the population over other combination, individual and control treatments. Maximum female nematode population $(27.00 / \mathrm{g}$ root) was noticed in control whereas the minimum (9.33) was observed in a combination treatment $\mathrm{T}_{11}$. Individual treatments viz., seed treatment $\left(\mathrm{T}_{1}\right)$, seedling treatments $\left(\mathrm{T}_{2}\right.$ and $\left.\mathrm{T}_{3}\right)$ and soil application treatments $\left(\mathrm{T}_{4}\right.$ and $\left.\mathrm{T}_{5}\right)$ significantly reduced the population registering $23.00,20.00,18.33,16.00$ and 15.33 females per gram root respectively over control $\mathrm{T}_{12}(27.00)$ (Table 2).

Results revealed significant reduction in gall formation by integrated treatments where the minimum number of galls per plant was noticed in $\mathrm{T}_{11}$ (13.67) followed by $\mathrm{T}_{10}(18.33), \mathrm{T}_{9}(18.67)$ and $\mathrm{T}_{8}(19.00)$ and the effect of these three treatments in reducing gall formation were statistically on par and the reduction over control $\mathrm{T}_{12}$ (39.00) in the above four treatments were 64.96, 52.99, 52.14 and 51.28 per cent respectively (Table 2).

Root gall index and root knot nematode damage evidenced maximum damage (4.0 root gall index and 50.00 per cent damage) whereas a combination treatment $\left(\mathrm{T}_{11}\right)$ exhibited the minimum damage $(2.7$ root gall index and 30.00 per cent damage) (Table 3 ). In integrated treatments, maximum egg mass parasitism was evidenced in $\mathrm{T}_{11}$ (60.00 per cent) which was significantly superior to their individual treatments $T_{3}\left(16.67\right.$ per cent) and $T_{5}(20.00$ per cent) and other combination treatments $\mathrm{T}_{6}(26.67$ per cent $), \mathrm{T}_{7}\left(26.67\right.$ per cent), $\mathrm{T}_{8}\left(36.67\right.$ per cent), $\mathrm{T}_{9}(33.33$ per cent) and $\mathrm{T}_{10}\left(40.00\right.$ per cent). Fruit yield was highest $(217.67 \mathrm{~g} /$ plant $)$ in a combination treatment $\mathrm{T}_{11}$, whereas the minimum yield was obtained in control plot which received no fungal inoculum (175.00 g). 
Comparing the individual treatments' and integrated treatments' effect, cumulative effect of individual treatments exerted additional yield in combination treatment (Table 3).

Table 3. Effect of different application methods of P. lilacinus on M. incognita damage, egg mass parasitism and yield of okra at Annamalainagar

\begin{tabular}{|c|c|c|c|c|c|}
\hline \multirow[b]{2}{*}{ Treatments } & \multirow[b]{2}{*}{ Gall index } & \multirow{2}{*}{$\begin{array}{c}\text { Root knot } \\
\text { damage (\%) }\end{array}$} & \multirow{2}{*}{$\begin{array}{c}\text { Egg mass } \\
\text { infection } \\
(\%)\end{array}$} & \multicolumn{2}{|c|}{ Yield/plant (g) } \\
\hline & & & & Fruit yield & $\begin{array}{c}\% \uparrow \downarrow \\
\text { OC }\end{array}$ \\
\hline $\mathrm{T}_{1}$ & 3.0 & 36.67 & $16.67 \pm 5.77^{\mathrm{d}}$ & $189.67 \pm 4.04^{g h}$ & 8.38 \\
\hline $\mathrm{T}_{2}$ & 3.3 & 50.00 & $20.00 \pm 10.00^{\mathrm{cd}}$ & $186.00 \pm 5.00^{\mathrm{n}}$ & 6.29 \\
\hline $\mathrm{T}_{3}$ & 3.0 & 36.67 & $16.67 \pm 5.77^{\mathrm{d}}$ & $192.33 \pm 4.04^{\mathrm{tg}}$ & 9.90 \\
\hline $\mathrm{T}_{4}$ & 3.0 & 36.67 & $23.33 \pm 11.55^{\mathrm{bcd}}$ & $193.00 \pm 1.73^{\mathrm{tg}}$ & 10.29 \\
\hline $\mathrm{T}_{5}$ & 3.0 & 30.00 & $20.00 \pm 17.32^{\mathrm{a}}$ & $196.33 \pm 2.08^{t}$ & 12.19 \\
\hline $\mathrm{T}_{6}$ & 3.0 & 36.67 & $26.67 \pm 15.28^{\mathrm{Dca}}$ & $196.33 \pm 0.58^{\mathrm{I}}$ & 12.19 \\
\hline $\mathrm{T}_{7}$ & 3.0 & 30.00 & $26.67 \pm 5.77^{\mathrm{bcd}}$ & $201.67 \pm 3.06^{\mathrm{e}}$ & 15.24 \\
\hline $\mathrm{T}_{8}$ & 3.0 & 30.00 & $36.67 \pm 5.77^{\mathrm{b}}$ & $205.67 \pm 2.52^{\mathrm{e}}$ & 17.52 \\
\hline $\mathrm{T}_{9}$ & 3.0 & 30.00 & $33.33 \pm 11.55^{\mathrm{bc}}$ & $204.67 \pm 4.51^{\mathrm{d}}$ & 16.95 \\
\hline $\mathrm{T}_{10}$ & 3.0 & 30.00 & $40.00 \pm 10.00^{b}$ & $212.67 \pm 4.73^{c}$ & 21.52 \\
\hline $\mathrm{T}_{11}$ & 2.7 & 30.00 & $60.00 \pm 10.00^{\mathrm{a}}$ & $217.67 \pm 6.66^{\mathrm{b}}$ & 24.38 \\
\hline $\mathrm{T}_{12}$ & 4.0 & 50.00 & 0.00 & $175.00 \pm 4.58^{\mathrm{a}}$ & \\
\hline S.Em \pm & & & 5.279 & 2.359 & \\
\hline C.D. $(P=0.05)$ & & & 10.947 & 4.892 & \\
\hline
\end{tabular}

Each figure is the mean of three replicates; in a column, means followed by common letter(s) are not significantly different $(P=0.05)$ by Least Significant Difference Test; DAP-Days after planting; \% $\uparrow \downarrow$ OC-per cent increase or decrease over control; $\mathrm{T}_{1}-P$. lilacinus seed treatment, $\mathrm{T}_{2}-P$. lilacinus seedling treatment $(5 \mathrm{~g} / 1$ water). $\mathrm{T}_{3}-P$. lilacinus seedling treatment $(10 \mathrm{~g} / 1$ water $), \mathrm{T}_{4}-P$. lilacinus soil application $(2.5 \mathrm{~kg} / \mathrm{acre}), \mathrm{T}_{5}-P$. lilacinus soil application (5.0 kg/acre), $\mathrm{T}_{6}-\mathrm{T}_{1}+\mathrm{T}_{4}, \mathrm{~T}_{7}-\mathrm{T}_{2}+\mathrm{T}_{4}, \mathrm{~T}_{8}-\mathrm{T}_{3}+\mathrm{T}_{4}, \mathrm{~T}_{9}-\mathrm{T}_{1}+\mathrm{T}_{5}, \mathrm{~T}_{10}-\mathrm{T}_{2}+\mathrm{T}_{5}, \mathrm{~T}_{11}-\mathrm{T}_{3}+\mathrm{T}_{5}$; and $\mathrm{T}_{12}$-Control; Data for treatments with zero means were omitted from the analysis of variance.

\subsection{Field Trial II}

Similar to first trial shoot length of okra plants registered in all treatments after 15 days of sowing did not show any significant difference, however fungal treatments evidenced increased shoot length at 60 and 90 days of sowing. Regarding root length, combination treatments $T_{11}$ and $T_{10}$ accounted 30.00 and $29.67 \mathrm{~cm}$ root length respectively with on par effect. Even though on par effect exist between the above two treatments, $\mathrm{T}_{11}$ exhibited significant difference over control and individual treatments. The same trend of irregular root weight was evidenced with combination treatments also, whereas the maximum root weight was noticed in the control (20.00g) (Table 4).

Data showed that nematode population in soil and roots were reduced significantly in the combination of seedling treatment $\left(T_{3}\right)$ and soil application treatment $\left(T_{5}\right)$ compared to control. Root knot nematode population in roots i.e., female and galls population in roots reduced drastically in combination treatments, particularly in $\mathrm{T}_{11}$ (3.67 females/g root and 12.00 galls/plant) compared to control $\mathrm{T}_{12}$ (16.67 females/g and root 36.33 galls/plant) (Table 5). Root gall index and root knot damage estimated from galls on roots disclosed similar view with lowest in $\mathrm{T}_{11}$ (root gall index 3.0 and root knot damage 23.33 per cent) and highest in control (root gall index 4.0 and root knot damage 50.00 per cent). Parasitism by P. lilacinus evidenced maximum in $\mathrm{T}_{10}(60.00$ per cent) followed by $\mathrm{T}_{11}$ (56.67 per cent) with on par effect. Relating all treatments, significant differences exist between combination and individual treatments with higher parasitism in a combination treatment $T_{11}$ than in individual treatments (Table 6). The maximum fruit yield was recorded in an associated treatment $\mathrm{T}_{11}(247.00 \mathrm{~g})$ which found to be significantly superior to their individual treatments $\mathrm{T}_{3}(203.33 \mathrm{~g})$ and $\mathrm{T}_{5}(216.33 \mathrm{~g})$ and also to control (187.33 g) (Table 6). 
Table 4. Influence of different application methods of P. lilacinus on the growth of okra infested with M.incognita at Vallambadugai

\begin{tabular}{|c|c|c|c|c|c|c|c|c|c|c|c|}
\hline \multirow{2}{*}{$\begin{array}{c}\text { Treatmen } \\
\text { ts }\end{array}$} & \multicolumn{5}{|c|}{ Shoot length $(\mathrm{cm})$} & \multicolumn{2}{|c|}{ Shoot weight (g) } & \multicolumn{2}{|c|}{ Root length $(\mathrm{cm})$} & \multicolumn{2}{|c|}{ Root weight } \\
\hline & Initial & $60 \mathrm{DAS}$ & $\% \uparrow \downarrow O C$ & $90 \mathrm{DAS}$ & $\% \uparrow \downarrow O C$ & $90 \mathrm{DAS}$ & $\% \uparrow \downarrow O C$ & $90 \mathrm{DAS}$ & $\% \uparrow \downarrow l 0 c$ & 90 DAS & $\% \uparrow \downarrow 0 \mathrm{C}$ \\
\hline $\mathrm{T}_{1}$ & $\begin{array}{c}16.67 \pm 0 \\
.58\end{array}$ & $\begin{array}{c}38.33 \pm 0.5 \\
8^{\mathrm{fg}}\end{array}$ & 1.76 & $\begin{array}{c}50.67 \pm 0.5 \\
8^{\text {gh }}\end{array}$ & 2.01 & $\begin{array}{c}45.33 \pm 3.5 \\
1^{\mathrm{c}}\end{array}$ & 0.01 & $\begin{array}{c}24.67 \pm 0.5 \\
8^{\text {efg }}\end{array}$ & -12.93 & $\begin{array}{c}15.67 \pm 0 . \\
58^{\mathrm{de}}\end{array}$ & -21.67 \\
\hline $\mathrm{T}_{2}$ & $\begin{array}{c}16.50 \pm 0 \\
.50\end{array}$ & $\begin{array}{c}39.33 \pm 0.5 \\
8^{\text {ef }}\end{array}$ & 4.42 & $\begin{array}{c}51.67 \pm 1.5 \\
3^{\mathrm{g}}\end{array}$ & 4.02 & $\begin{array}{c}46.33 \pm 3.5 \\
1^{\mathrm{bc}}\end{array}$ & 2.21 & $\begin{array}{c}24.33 \pm 0.5 \\
8^{\mathrm{fg}}\end{array}$ & -14.11 & $\begin{array}{c}16.00 \pm 1 . \\
00^{\mathrm{cde}}\end{array}$ & -20.00 \\
\hline $\mathrm{T}_{3}$ & $\begin{array}{c}16.83 \pm 0 \\
.29\end{array}$ & $\begin{array}{c}39.33 \pm 0.5 \\
8^{\text {ef }}\end{array}$ & 4.42 & $\begin{array}{c}52.00 \pm 1.0 \\
0^{\mathrm{g}}\end{array}$ & 4.69 & $\begin{array}{c}47.33 \pm 3.5 \\
1^{\text {bc }}\end{array}$ & 4.42 & $\begin{array}{c}23.00 \pm 1.0 \\
0^{\mathrm{g}}\end{array}$ & -18.81 & $\begin{array}{c}14.67 \pm 0 . \\
58^{\mathrm{e}}\end{array}$ & -26.67 \\
\hline $\mathrm{T}_{4}$ & $\begin{array}{c}17.67 \pm 1 \\
.53\end{array}$ & $\begin{array}{c}40.33 \pm 0.5 \\
8^{\text {de }}\end{array}$ & 7.07 & $\begin{array}{c}53.67 \pm 0.5 \\
8^{\mathrm{f}}\end{array}$ & 8.05 & $\begin{array}{c}47.33 \pm 3.5 \\
1^{\text {bc }}\end{array}$ & 4.42 & $\begin{array}{c}26.33 \pm 0.5 \\
8^{\text {cde }}\end{array}$ & -7.05 & $\begin{array}{c}16.33 \pm 0 . \\
58^{\text {b-e }}\end{array}$ & -18.33 \\
\hline $\mathrm{T}_{5}$ & $\begin{array}{c}17.00 \pm 0 \\
.00\end{array}$ & $\begin{array}{c}41.33 \pm 0.5 \\
8^{\text {cd }}\end{array}$ & 9.72 & $\begin{array}{c}54.33 \pm 0.5 \\
8^{\text {ef }}\end{array}$ & 9.39 & $\begin{array}{c}48.33 \pm 3.5 \\
1^{\text {bc }}\end{array}$ & 6.63 & $\begin{array}{c}27.33 \pm 0.5 \\
8^{\text {bc }}\end{array}$ & -3.52 & $\begin{array}{c}14.33 \pm 0 . \\
58^{\mathrm{e}}\end{array}$ & -28.33 \\
\hline $\mathrm{T}_{6}$ & $\begin{array}{c}17.83 \pm 0 \\
.29\end{array}$ & $\begin{array}{c}41.67 \pm 0.5 \\
8^{\mathrm{bc}}\end{array}$ & 10.61 & $\begin{array}{c}55.33 \pm 0.5 \\
8^{\text {cde }}\end{array}$ & 11.40 & $\begin{array}{c}48.00 \pm 1.0 \\
0^{\mathrm{bc}}\end{array}$ & 5.89 & $\begin{array}{c}26.67 \pm 0.5 \\
8^{\text {cd }}\end{array}$ & -5.87 & $\begin{array}{c}15.33 \pm 0 . \\
58^{\mathrm{de}}\end{array}$ & -23.33 \\
\hline $\mathrm{T}_{7}$ & $\begin{array}{c}17.33 \pm 0 \\
.58\end{array}$ & $\begin{array}{c}42.00 \pm 1.0 \\
0^{\text {bc }}\end{array}$ & 11.49 & $\begin{array}{c}55.00 \pm 1.0 \\
0^{\text {def }}\end{array}$ & 10.73 & $\begin{array}{c}48.33 \pm 0.5 \\
8^{\text {bc }}\end{array}$ & 6.63 & $\begin{array}{c}28.67 \pm 2.0 \\
8^{\text {ab }}\end{array}$ & 1.19 & $\begin{array}{c}14.67 \pm 2 . \\
52^{\mathrm{e}}\end{array}$ & -26.67 \\
\hline $\mathrm{T}_{8}$ & $\begin{array}{c}16.83 \pm 0 \\
.29\end{array}$ & $\begin{array}{c}42.33 \pm 0.5 \\
8^{\mathrm{abc}}\end{array}$ & 12.38 & $\begin{array}{c}57.33 \pm 0.5 \\
8^{\text {ab }}\end{array}$ & 15.43 & $\begin{array}{c}49.33 \pm 3.5 \\
1^{\mathrm{ab}}\end{array}$ & 8.83 & $\begin{array}{c}27.33 \pm 2.0 \\
8^{\text {bc }}\end{array}$ & -3.52 & $\begin{array}{c}16.33 \pm 2 . \\
08^{\text {b-e }}\end{array}$ & -18.33 \\
\hline $\mathrm{T}_{9}$ & $\begin{array}{c}17.67 \pm 1 \\
.53\end{array}$ & $\begin{array}{c}42.67 \pm 0.5 \\
8^{\text {ab }}\end{array}$ & 13.26 & $\underset{0^{\text {bed }}}{56.00 \pm 1.0}$ & 12.74 & $\begin{array}{c}48.33 \pm 3.5 \\
1^{\text {bc }}\end{array}$ & 6.63 & $\begin{array}{c}29.00 \pm 1.0 \\
0^{\text {ab }}\end{array}$ & 2.36 & $\begin{array}{c}17.67 \pm 1 . \\
53^{\mathrm{a}-\mathrm{d}}\end{array}$ & -11.67 \\
\hline $\mathrm{T}_{10}$ & $\begin{array}{c}17.00 \pm 0 \\
.00\end{array}$ & $\begin{array}{c}42.67 \pm 0.5 \\
8^{\text {ab }}\end{array}$ & 13.26 & $\begin{array}{c}56.67 \pm 0.5 \\
8^{\text {bc }}\end{array}$ & 14.09 & $\begin{array}{c}49.33 \pm 3.5 \\
1^{\mathrm{ab}}\end{array}$ & 8.83 & $\begin{array}{c}29.67 \pm 0.5 \\
8^{a}\end{array}$ & 4.72 & $\begin{array}{c}18.67 \pm 1 . \\
53^{\mathrm{ab}}\end{array}$ & -6.67 \\
\hline $\mathrm{T}_{11}$ & $\begin{array}{c}16.67 \pm 0 \\
.58\end{array}$ & $\begin{array}{c}43.33 \pm 0.5 \\
8^{\mathrm{a}}\end{array}$ & 15.03 & $\begin{array}{c}58.00 \pm 1.0 \\
0^{\mathrm{a}}\end{array}$ & 16.77 & $\begin{array}{c}52.33 \pm 3.5 \\
1^{\mathrm{a}}\end{array}$ & 15.45 & $\begin{array}{c}30.00 \pm 1.0 \\
0^{\mathrm{a}}\end{array}$ & 5.89 & $\begin{array}{c}18.33 \pm 2 . \\
52^{\mathrm{abc}}\end{array}$ & -8.33 \\
\hline $\mathrm{T}_{12}$ & $\begin{array}{c}16.83 \pm 0 \\
.29\end{array}$ & $\begin{array}{c}37.67 \pm 1.5 \\
3^{\mathrm{g}}\end{array}$ & & $\begin{array}{c}49.67 \pm 1.5 \\
3^{\mathrm{h}}\end{array}$ & & $\begin{array}{c}45.33 \pm 0.5 \\
8^{\mathrm{c}}\end{array}$ & & $\begin{array}{c}28.33 \pm 3.7 \\
9^{\mathrm{f}}\end{array}$ & & $\begin{array}{c}20.00 \pm 2 . \\
65^{\mathrm{a}}\end{array}$ & \\
\hline S. $\mathrm{Em} \pm$ & NS & 0.607 & & 0.777 & & 1.606 & & 0.928 & & 1.285 & \\
\hline $\begin{array}{c}\text { C.D. } \\
(P=0.05)\end{array}$ & & 1.259 & & 1.612 & & 3.331 & & 1.925 & & 2.665 & \\
\hline
\end{tabular}

Each value is an average of three replicates; in a column, means followed by common letter(s) are not significantly different $(P=0.05)$ by Least Significant Difference Test; DAP-Days after planting; \% $\uparrow \downarrow$ OC-per cent increase or decrease over control; NS-No significance at $P=0.05 ; \mathrm{T}_{1}-P$. lilacinus seed treatment, $\mathrm{T}_{2}-P$. lilacinus seedling treatment $\left(5 \mathrm{~g} / 1\right.$ water). $\mathrm{T}_{3}-P$. lilacinus seedling treatment $(10 \mathrm{~g} / \mathrm{l}$ water $), \mathrm{T}_{4}-P$. lilacinus soil application (2.5 kg/acre), $\mathrm{T}_{5}-P$. lilacinus soil application (5.0 kg/acre), $\mathrm{T}_{6}-\mathrm{T}_{1}+\mathrm{T}_{4}, \mathrm{~T}_{7}-\mathrm{T}_{2}+\mathrm{T}_{4}, \mathrm{~T}_{8}-\mathrm{T}_{3}+\mathrm{T}_{4}, \mathrm{~T}_{9}-\mathrm{T}_{1}+\mathrm{T}_{5}$, $\mathrm{T}_{10}-\mathrm{T}_{2}+\mathrm{T}_{5}, \mathrm{~T}_{11}-\mathrm{T}_{3}+\mathrm{T}_{5}$; and $\mathrm{T}_{12}$-Control.

Table 5. Effect of different application methods of P. lilacinus on population density and gall formation of $M$. incognita in okra at Vallambadugai

\begin{tabular}{|c|c|c|c|c|c|c|c|c|}
\hline \multirow[b]{2}{*}{ Treatments } & \multicolumn{4}{|c|}{ Juvenile population (100 cc soil) } & \multicolumn{2}{|c|}{ Galls/plant } & \multicolumn{2}{|c|}{ Females/g root } \\
\hline & $60 \mathrm{DAS}$ & $\begin{array}{c}\% \uparrow \downarrow \\
\text { OC }\end{array}$ & 90 DAS & $\begin{array}{c}\% \uparrow \downarrow \\
\text { OC }\end{array}$ & $90 \mathrm{DAS}$ & $\begin{array}{c}\% \uparrow \downarrow \\
\text { OC }\end{array}$ & 90 DAS & $\begin{array}{c}\% \uparrow \downarrow \\
\text { OC }\end{array}$ \\
\hline $\mathrm{T}_{1}$ & $217.00 \pm 3.61^{\mathrm{e}}$ & -5.93 & $202.67 \pm 4.62^{\mathrm{fg}}$ & -5.59 & $25.00 \pm 1.00^{\mathrm{e}}$ & -31.19 & $9.67 \pm 0.58^{\mathrm{f}}$ & -42.01 \\
\hline $\mathrm{T}_{2}$ & $209.00 \pm 3.61^{\mathrm{de}}$ & -9.39 & $193.00 \pm 3.61^{\mathrm{ef}}$ & -10.09 & $24.00 \pm 6.08^{\mathrm{de}}$ & -33.94 & $9.33 \pm 0.58^{\mathrm{ef}}$ & -44.01 \\
\hline $\mathrm{T}_{3}$ & $201.00 \pm 5.00^{\mathrm{d}}$ & -12.86 & $188.33 \pm 3.06^{\text {def }}$ & -12.27 & $25.67 \pm 5.51^{\mathrm{e}}$ & -29.35 & $8.33 \pm 0.58^{\text {def }}$ & -50.01 \\
\hline $\mathrm{T}_{4}$ & $190.00 \pm 1.00^{\mathrm{c}}$ & -17.63 & $180.33 \pm 2.52^{\text {cde }}$ & -16.00 & $21.67 \pm 3.06^{\text {cde }}$ & -40.36 & $7.67 \pm 0.58^{\mathrm{c}-\mathrm{f}}$ & -54.01 \\
\hline $\mathrm{T}_{5}$ & $177.67 \pm 4.73^{b}$ & -22.98 & $174.33 \pm 5.86^{\mathrm{cd}}$ & -18.79 & $20.33 \pm 2.52^{\mathrm{b}-\mathrm{e}}$ & -44.03 & $6.67 \pm 0.58^{\mathrm{bcd}}$ & -60.01 \\
\hline $\mathrm{T}_{6}$ & $188.00 \pm 5.29^{\mathrm{c}}$ & -18.50 & $175.33 \pm 5.86^{\mathrm{cd}}$ & -18.32 & $18.33 \pm 2.89^{\text {bcd }}$ & -49.54 & $7.33 \pm 0.58^{\text {cde }}$ & -56.01 \\
\hline $\mathrm{T}_{7}$ & $178.33 \pm 4.93^{b}$ & -22.69 & $166.67 \pm 10.69^{\mathrm{bc}}$ & -22.36 & $18.00 \pm 2.00^{\mathrm{bc}}$ & -50.45 & $6.33 \pm 1.15^{\mathrm{bcd}}$ & -62.01 \\
\hline $\mathrm{T}_{8}$ & $174.67 \pm 3.79^{b}$ & -24.28 & $165.67 \pm 6.11^{\mathrm{bc}}$ & -22.83 & $16.67 \pm 2.52^{\mathrm{abc}}$ & -54.12 & $5.67 \pm 1.15^{\mathrm{abc}}$ & -66.01 \\
\hline $\mathrm{T}_{9}$ & $177.67 \pm 4.16^{\mathrm{b}}$ & -22.98 & $157.67 \pm 28.01^{\mathrm{ab}}$ & -26.55 & $9.00 \pm 2.65^{\mathrm{bc}}$ & -75.23 & $5.67 \pm 1.53^{\mathrm{abc}}$ & -66.01 \\
\hline $\mathrm{T}_{10}$ & $172.67 \pm 5.51^{b}$ & -25.15 & $155.00 \pm 7.81^{\mathrm{ab}}$ & -27.80 & $15.67 \pm 1.53^{\mathrm{ab}}$ & -56.88 & $4.67 \pm 1.53^{\mathrm{ab}}$ & -72.01 \\
\hline $\mathrm{T}_{11}$ & $151.00 \pm 1.00^{\mathrm{a}}$ & -34.54 & $145.33 \pm 4.93^{\mathrm{a}}$ & -32.30 & $12.00 \pm 2.65^{\mathrm{a}}$ & -66.97 & $3.67 \pm 0.58^{\mathrm{a}}$ & -78.00 \\
\hline $\mathrm{T}_{12}$ & $230.67 \pm 11.06^{\mathrm{f}}$ & & $214.67 \pm 4.16^{\mathrm{g}}$ & & $36.33 \pm 5.03^{\mathrm{f}}$ & & $16.67 \pm 3.06^{\mathrm{g}}$ & \\
\hline S. $E m \pm$ & 0.146 & & 0.311 & & 0.304 & & 1.034 & \\
\hline $\begin{array}{c}\text { C.D. } \\
(P=0.05)\end{array}$ & 0.302 & & 0.644 & & 0.630 & & 2.144 & \\
\hline
\end{tabular}

Initial juvenile population-302.67/100cc soil; each value is an average of three replicates; in a column, means 
followed by common letter(s) are not significantly different $(P=0.05)$ by Least Significant Difference Test; DAP-Days after planting; \% $\uparrow \downarrow$ OC-per cent increase or decrease over control; $\mathrm{T}_{1}-P$. lilacinus seed treatment, $\mathrm{T}_{2}-P$. lilacinus seedling treatment $\left(5 \mathrm{~g} / 1\right.$ water). $\mathrm{T}_{3}-P$. lilacinus seedling treatment $(10 \mathrm{~g} / 1 \mathrm{water}), \mathrm{T}_{4}-P$. lilacinus soil application (2.5 kg/acre), $\mathrm{T}_{5}-P$. lilacinus soil application (5.0 kg/acre), $\mathrm{T}_{6}-\mathrm{T}_{1}+\mathrm{T}_{4}, \mathrm{~T}_{7}-\mathrm{T}_{2}+\mathrm{T}_{4}, \mathrm{~T}_{8}-\mathrm{T}_{3}+\mathrm{T}_{4}, \mathrm{~T}_{9}$ $-\mathrm{T}_{1}+\mathrm{T}_{5}, \mathrm{~T}_{10}-\mathrm{T}_{2}+\mathrm{T}_{5}, \mathrm{~T}_{11}-\mathrm{T}_{3}+\mathrm{T}_{5}$; and $\mathrm{T}_{12}$-Control

Table 6. Effect of different application methods of P. lilacinus on M. incognita damage, egg mass parasitism and yield of okra at Vallambadugai

\begin{tabular}{cccccc}
\hline \multirow{2}{*}{ Treatments } & \multirow{2}{*}{ Gall index } & $\begin{array}{c}\text { Root knot damage } \\
(\%)\end{array}$ & $\begin{array}{c}\text { Egg mass infection } \\
(\%)\end{array}$ & \multicolumn{2}{c}{ Yield/plant $(\mathrm{g})$} \\
\cline { 5 - 6 } & & 36.67 & $26.67 \pm 5.77^{\mathrm{c}}$ & $200.00 \pm 4.36^{\mathrm{g}}$ & $\% \uparrow \downarrow$ OC \\
\hline $\mathrm{T}_{1}$ & 3.0 & 36.67 & $33.33 \pm 5.77^{\mathrm{bc}}$ & $198.33 \pm 5.51^{\mathrm{g}}$ & 5.87 \\
$\mathrm{~T}_{2}$ & 3.3 & 36.67 & $33.33 \pm 11.55^{\mathrm{c}}$ & $203.33 \pm 3.51^{\mathrm{fg}}$ & 8.54 \\
$\mathrm{~T}_{3}$ & 3.0 & 30.00 & $33.33 \pm 5.77^{\mathrm{bc}}$ & $208.33 \pm 3.51^{\mathrm{f}}$ & 11.21 \\
$\mathrm{~T}_{4}$ & 3.0 & 30.00 & $33.33 \pm 5.77^{\mathrm{bc}}$ & $216.33 \pm 5.69^{\mathrm{de}}$ & 15.48 \\
$\mathrm{~T}_{5}$ & 3.0 & 30.00 & $43.33 \pm 11.55^{\mathrm{abc}}$ & $220.67 \pm 1.53^{\mathrm{cd}}$ & 17.80 \\
$\mathrm{~T}_{6}$ & 3.0 & 30.00 & $33.33 \pm 5.77^{\mathrm{bc}}$ & $225.67 \pm 4.04^{\mathrm{bc}}$ & 20.46 \\
$\mathrm{~T}_{7}$ & 3.0 & 30.00 & $46.67 \pm 15.28^{\mathrm{abc}}$ & $230.33 \pm 4.16^{\mathrm{b}}$ & 22.96 \\
$\mathrm{~T}_{8}$ & 3.0 & 30.00 & $53.33 \pm 25.17^{\mathrm{ab}}$ & $226.67 \pm 3.06^{\mathrm{bc}}$ & 21.00 \\
$\mathrm{~T}_{9}$ & 3.0 & 30.00 & $60.00 \pm 17.32^{\mathrm{a}}$ & $233.67 \pm 4.73^{\mathrm{b}}$ & 24.74 \\
$\mathrm{~T}_{10}$ & 3.0 & 23.33 & $56.67 \pm 5.77^{\mathrm{a}}$ & $247.00 \pm 6.08^{\mathrm{a}}$ & 31.85 \\
$\mathrm{~T}_{11}$ & 3.0 & 50.00 & 0.00 & $187.33 \pm 8.74^{\mathrm{h}}$ & \\
$\mathrm{T}_{12}$ & 4.0 & & 5.820 & 4.016 & \\
$\mathrm{~S} . \mathrm{Em} \pm$ & & & 12.070 & 8.329 & \\
C.D. $(P=0.05)$ & & & & &
\end{tabular}

Each figure is the mean of three replicates; in a column, means followed by common letter(s) are not significantly different $(\mathrm{P}=0.05)$ by Least Significant Difference Test; DAP-Days after planting; \% $\uparrow \downarrow$ OC-per cent increase or decrease over control; T1-P. lilacinus seed treatment, T2-P. lilacinus seedling treatment $(5 \mathrm{~g} / 1$ water). T3-P. lilacinus seedling treatment $(10 \mathrm{~g} / 1$ water $)$, T4-P. lilacinus soil application $(2.5 \mathrm{~kg} / \mathrm{acre}), \mathrm{T} 5-P$. lilacinus soil application (5.0 kg/acre), T6-T1+T4, T7-T2+T4, T8-T3+T4, T9 -T1+T5, T10-T2+T5, T11-T3+T5; and T12-Control; ; Data for treatments with zero means were omitted from the analysis of variance

In the present study, integration of different methods of application of P. lilacinus viz., seed treatment, seedling treatments and soil application treatments under field conditions exhibited an increase in plant growth parameters (shoot length, shoot weight, root length and root weight) in the fields trial conducted both places. Shoot length documented after 60 and 90 days after sowing among individual treatments demonstrated higher values in soil application treatment (5.0 kg/acre) compared to seed treatment and seedling treatment. Similarly Shanmugapriya and Kumar (2006a) reported that seedling application was found better in increasing plant growth parameters and reducing nematode population compared to seedling treatment, seed treatment and control in tomato.These findings are in agreement with Khan \& Saxena (1996) who reported that $P$. lilacinus united with organic nutrients produced better environment to the crop and resulted an increased plant growth.

Increased egg infection by $P$. lilacinus results in diminution of nematode population, which was noticed in this study, even the individual seed treatment, seedling treatment and soil application treatments and their combination treatments In the present investigations, egg mass parasitism accounted through fungal mycelium was higher in combination treatments principally in the mixing of seedling treatment $(10 \mathrm{~g} / \mathrm{l})+$ soil application treatment $(5.0 \mathrm{~kg} / \mathrm{acre})(60.00 \%)$ and when matched with their individual treatments which was 72.21 and 66.66 percent additional parasitism than their individual treatments respectively. These reports are in concurrence with that of Khan and Husain (1988) who asserted that increased P. lilacinus inclusion exerted an increased egg mass parasitism. Similarly, Ibrahim et al. (1987) and Walia et al. (1991) also reported increased egg mass parasitism with the increase of fungal inoculum. The improvement in biometric characters and resulted decline in nematode population displayed a direct impact on yield of okra in terms of weight of fruits. Fruit yield in seedling treatment $(10 \mathrm{~g} / \mathrm{l}$ water $)+$ soil application treatment $(5.0 \mathrm{~kg} / \mathrm{acre})$ combination was maximum with 24.38 per cent more yield than in control. These treatments are economic, environmentally safe and sustainable in improving the vigour of plants and also the yield (Bhagawati et al., 2007)

Taking into account the root weight assessment, the whole fungal treatments neither lone method of application nor integrated application significantly increased the root weight, mainly in an integrated treatment involving seedling treatment $(10 \mathrm{~g} / \mathrm{l}$ water) and soil application treatment $(5.0 \mathrm{~kg} /$ acre $)$ the root weight was elevated 
between them, on the other hand, maximum root weight was recorded in control which was 8.33 per cent more weight than in integrated treatment. The increased root weight may be due to the greater gall formation by $M$. incognita, which increased the size of galls resulting in higher root weight as suggested by Khan et al. (2001). This result is in contrast to the reports of Pandey and Dwivedi (2001) and Dhawan et al. (2004) who recorded maximum root weight in P. lilacinus applied treatments compared to control due to fungal treatment's effect on root which resulted nematode population in roots.

The elective effect of $P$. lilacinus was attributed to the toxic, and enzymatic principles such as acetic acid and proteolytic and chitinolytic enzymes released during fungal interaction with nematode which might cause killing/inhibiting the $M$. incognita directly or indirectly promoting plant growth and ultimately enhancing yield component of okra (Mahapatra \& Sahani, 2007). In both the trials, seed treatment, seedling treatment and soil application treatments and their combination treatments significantly increased the plant growth characters, yield, and egg mass infection except root weight and reduced the nematode population and these results are in line with the reports of Shanmugapriya and Kumar (2006b) in tomato and Gomathi et al. (2006) in brinjal.

\section{Conclusion}

This research showed that shoot length, shoot weight and root length were significantly increased in mixture treatments compared to individual treatments in field evaluation of okra in both Annamalainagar and Vallambadugai regions. In particular, the combination of seedling treatment $(10 \mathrm{~g} / \mathrm{l}$ water $)+$ soil application treatment (5.0 kg/acre) documented maximum shoot length (60 and 90 DAS), shoot weight (90 DAS) and root length (90 DAS) and they were positively correlated with fruit yield of okra.

\section{Acknowledgements}

This research was supported by Department of Entomology, Faculty of Agriculture, Annamalai University. Many thanks to post graduate and research scholars from Department of Entomology.

\section{References}

Ahmad, S. F., \& Khan, T. A. (2004).Management of root knot nematode, Meloidogyne incognita by integration of Paecilomyces lilacinus with organic materials in chilli. Archives Phytopathol Plant Protec, 37 (1), 35-40. http://dx.doi.org/10.1080/03235400310001631918

Bhagawati, B., Das, B.C. andSinha, A. K. (2007). Interaction of Meloidogyne incognita and Rhizoctoniasolani on okra.Ann Pl ProtecSci, 15(2), 533-535.

Brand, D., Roussos, S., Pandey, A., Zilioli, P. C., Pohl, J. \& Soccol, C. R. (2004). Development of a bio-nematicide with Paecilomyces lilacinus to control Meloidogyne incognita. Appl Biochem Biotechnol, 118 (1-3), 81-88. http://dx.doi.org/10.1385/ABAB:118:1-3:081

Cabanillas, E., and Barker, K. R. (1989). Impact of Paecilomyces lilacinus inoculum level and application time on control of Meloidogyne incognita on tomato. J. Nematol, 21, 115-120.

Devrajan, K., \& Rajendran, G. (2002). Effect of fungal egg parasite, Paecilomyces lilacinus (Thom.) Samson on Meloidogyne incognita in banana. Indian J. Nematol, 32 (1), 88-90.

Dhawan, S. C., Narayana, R., \& Babu, N. P. (2004). Bio-management of root knot nematode, Meloidogyne incognita in okra by Paecilomyces lilacinus. Ann. Pl. Protec. Sci., 12(2), 356-359.

Gomathi, C., Kumar, S., \& Subramanian, S. (2006). Effective dose and methods of delivery of Pasteuria penetrans against root knot nematode in brinjal. Ann. Pl. Protec. Sci., 14(2), 452-455.

Gomez, K. A., \& Gomez, A. A. (1976). Statistical procedure for agricultural research with emphasis on rice (p. 294). Las Banos, Philippines: International Rice Research Institute

Hasan, N. (2004). Evaluation of native strain of Paecilomyces lilacinus against Meloidogyne incognita in cowpea followed by leucerne. Ann. Pl. Protec. Sci., 12, 121-124.

Ibrahim, I. K. A., Rezk, M. A., El-Sadedy, M. A. \& Ibrahim, A. A. M. (1987). Control of Meloidogyne incognita on corn, tomato and okra with Paecilomyces lilacinus and the nematicide aldicarb. Nematol Medit, 15, 265-268.

Jain, R. K., Mathur, K. N. \& Singh, R. V. (2007). Estimation of losses due to plant parasitic nematodes on different crops. Indian J. Nematol, 37(2), 219-221.

Jatala, P., Kaltenbach, R., Bocangel, M., Devaux, A. J. \& Campos, R. (1980). Field application of Paecilomyces lilacinus for controlling Meloidogyne incognita on potatoes. J. Nematol, 12, 226-227. 
Khan, A. A., \& Khan, M. W. (1990). Infestation, distribution pattern and identity of root knot nematode associated with vegetable crops in the district of Meerut division in UP India. Indian J Nematol, 20, 67-75.

Khan, H. U., Ahmad, R., Ahmad, W., Khan, S. M., \& Aslam Khan, M. (2001). Evaluation of the combined effects of Paecilomyces lilacinus and Trichoderma harzianum against root knot disease of tomato. J. Biol. Sci., 1(3), 139-142. http://dx.doi.org/10.3923/jbs.2001.139.142

Khan, T. A., \& Husain, S. A. (1988). Studies on the efficacy of Paecilomyces lilacinus as biocontrol agent against a disease complex caused by the interaction of Rotylenchulus reniformis, Meloidogyne incognita and Rhizoctonia solani on cowpea. Nematol Medit, 16, 229-231.

Khan, T. A., \& Saxena, S. K. (1996). Comparative efficacy of Paecilomyces lilacinus in the control of Meloidogyne spp. and Rotylenchulus reniformis on tomato. Pak J. Nematol, 13, 111-116.

Khan, T. A., Azam, M. A., \& Husain, S. I. (1985). Effect of fungal filtrates of Aspergillus niger and Rhizoctinia solani on penetration and development of root knot nematode and the plant growth of tomato var. Marglobe. Indian J. Nematol, 14, 106-109.

Kiewnick, S., \& Sikora, R. A. (2006). Biological control of the root knot nematode Meloidogyne incognita by Paecilomyces lilacinus strain 251. Biological Control, 38(2), 179-187. http://dx.doi.org/10.1016/j.biocontrol.2005.12.006

Mahapatra, S. N., \& Sahani, N. R. (2007). Toxicity of some fungal filtrates on the development of Meloidogyne incognita in brinjal roots. Indian J. Nematol, 37 (1), 99-100.

Mojumder, V., Pankaj, J., Chawla, G., \& Singh, J. (2004). Effect of urea coated with neem formulations on root knot and reniform nematodes in okra. Indian J. Nematol, 34(1), 60-63.

Pandey, R. C., \& Dwivedi, B. K. (2001). Study on the effect of different biocontrol agent against root knot disease of brinjal. Current Nematol, 12(1,2), 73-74.

Rangaswamy, R. (1995). A text book of agricultural statistics. New Delhi: New Age International Publishers Limited, Wiley Eastern Limited.

Shanmugapriya, M., \& Kumar, S. (2006a). Studies on the method of application of the fungus, Paecilomyces lilacinus against root knot nematode on tomato. Indian J. Nematol, 36(1), 23-26.

Shanmugapriya, M., \& Kumar, S. (2006b). Dose optimization of Paecilomyces lilacinus for the control of Meloidogyne incognita on tomato. Indian J. Nematol, 36(1), 27-31.

Sivakumar, C. V., Palaniswamy, S., \& Naganathan, T. G. (1976). Persistence of nematicidal activities in seed treatment with carbofuran and aldicarb sulfone. Indian J. Nematol, 6, 106-108.

Swarup, G. (1962). Root knot of vegetable.Prevalence of Meloidogyne spp. in soil and vegetable crops. Indian Phytopathol, 15, 228-236.

Tiyagi, S. A., \& Ajaz, S. (2004). Biological control of plant parasitic nematodes associated with chickpea using oil cakes and Paecilomyces lilacinus. Indian J. Nematol, 34(1), 44-48.

Walia, R. K., Bansal, R. K., \& idogyne javanica on okra. Nematol Medit, 19, 247-249.

Zaki, F. A., \& Bhatti, D. S. (1999). Effect of Castor (Ricinus communis) and the biocontrol fungus Paecilomyces

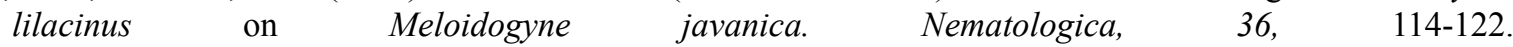
http://dx.doi.org/10.1163/002925990X00086 